\section{Silhouette Coherence for Camera Calibration under Circular Motion}

\author{
Carlos Hernández, Member, IEEE, Francis Schmitt, \\ and Roberto Cipolla, Member, IEEE
}

\begin{abstract}
We present a new approach to camera calibration as a part of a complete and practical system to recover digital copies of sculpture from uncalibrated image sequences taken under turntable motion. In this paper, we introduce the concept of the silhouette coherence of a set of silhouettes generated by a 3D object. We show how the maximization of the silhouette coherence can be exploited to recover the camera poses and focal length. Silhouette coherence can be considered as a generalization of the well-known epipolar tangency constraint for calculating motion from silhouettes or outlines alone. Further, silhouette coherence exploits all the geometric information encoded in the silhouette (not just at epipolar tangency points) and can be used in many practical situations where point correspondences or outer epipolar tangents are unavailable. We present an algorithm for exploiting silhouette coherence to efficiently and reliably estimate camera motion. We use this algorithm to reconstruct very high quality 3D models from uncalibrated circular motion sequences, even when epipolar tangency points are not available or the silhouettes are truncated. The algorithm has been integrated into a practical system and has been tested on more than 50 uncalibrated sequences to produce high quality photo-realistic models. Three illustrative examples are included in this paper. The algorithm is also evaluated quantitatively by comparing it to a state-of-the-art system that exploits only epipolar tangents.
\end{abstract}

Index Terms-Silhouette coherence, epipolar tangency, image-based visual hull, focal length estimation, circular motion, 3D modeling.

\section{INTRODUCTION}

COMPUTER vision techniques are becoming increasingly popular for the acquisition of high quality 3D models from image sequences. This is particularly true for the digital archiving of cultural heritage, such as museum objects and their 3D visualization, making them available to people without physical access.

Recently, a number of promising multiview stereo reconstruction techniques have been presented that are now able to produce very dense and textured 3D models from calibrated images. These are typically optimized to be consistent with stereo cues in multiple images by using space carving [1], deformable meshes [2], volumetric optimization [3], or depth maps [4].

The key to making these systems practical is that they should be usable by a nonexpert in computer vision such as a museum photographer, who is only required to take a sequence of high quality still photographs. In practice, a particularly convenient way to acquire the photographs is to use a circular motion or turntable setup (see Fig. 1 for two examples), where the object is rotated in front of a fixed, but uncalibrated camera. Camera calibration is thus a major obstacle in the model acquisition pipeline. For many museum objects, between 12 and 72 images are typically acquired and automatic camera calibration is essential.

Among all the available camera calibration techniques, pointbased methods are the most popular (see [5] for a review and [6] for a

- C. Hernández and R. Cipolla are with the Department of Engineering, MI Lab, Room 426 (Fallside Laboratory), University of Cambridge, Cambridge, CB2 1PZ, UK. E-mail: \{ch394, cipolla\}@eng.cam.ac.uk.

- F. Schmitt is with GET/ENST Télécom Paris, CNRS UMR 5141, Signal and Image Processing Department, Room C204, 46 rue Barrault, 75634 Paris Cedex 13, France. E-mail: francis.schmitt@enst.fr.

Manuscript received 27 Feb. 2006; revised 6 July 2006; accepted 10 July 2006; published online 13 Dec. 2006.

Recommended for acceptance by M. Pollefeys.

For information on obtaining reprints of this article, please send e-mail to: tpami@computer.org, and reference IEEECS Log Number TPAMI-0189-0206. state-of-the-art implementation). These rely on the presence of feature points on the object surface and can provide very accurate camera estimation results. Unfortunately, especially in case of manmade objects and museum artifacts, feature points are not always available or reliable (see the example in Fig. 1b). For such sequences, there exist alternative algorithms that use the object outline or silhouette as the only reliable image feature, exploiting the notion of epipolar tangents and frontier points [7], [8], [9] (see [10] for a review). In order to give accurate results, these methods require very good quality silhouettes, making their integration in a practical system difficult. For the particular case of turntable motion, the silhouette segmentation bottleneck is the separation of the object from the turntable. A common solution is to clip the silhouettes (see example in Fig. 1b). Another instance of truncated silhouettes occurs when acquiring a small region of a bigger object (see Fig. 1a).

We present a new approach to silhouette-based camera motion and focal length estimation that exploits the notion of multiview silhouette coherence. In brief, we exploit the rigidity property of 3D objects to impose the key geometric constraint on their silhouettes, namely, that there must exist a 3D object that could have generated these silhouettes. For a given set of silhouettes and camera projection matrices, we are able to quantify the agreement of both the silhouettes and the projection matrices, i.e., how much of the silhouettes could have been generated by a real object given those projection matrices. Camera estimation is then seen as an optimization step where silhouette coherence is treated as a function of the camera matrices that has to be maximized. The proposed technique extends previous silhouette-based methods and can deal with partial or truncated silhouettes, where the estimation and matching of epipolar tangents can be very difficult or noisy. It also exploits more information than is available just at epipolar tangency points. It is especially convenient when combined with 3D object modeling techniques that already fuse silhouettes with additional cues, as in [2], [3].

This paper is organized as follows: In Section 2, we review the literature. In Section 3, we state our problem formulation. In Section 4, we introduce the concept of silhouette coherence. In Section 5 ,we describe the actual algorithm for camera calibration. In Section 6, we illustrate the accuracy of the method and show some high quality reconstructions.

\section{Previous Work}

Many algorithms for camera motion estimation and autocalibration have been reported [5]. They typically rely on correspondences between the same features detected in different images. For the particular case of circular motion, the methods of [11] and [12] work well when the images contain enough texture to allow a robust detection of their features. An alternative is to exploit silhouettes. Silhouettes have already been used for camera motion estimation using the notion of epipolar tangency points [7], [8], [13], i.e., points on the silhouette contours in which the tangent to the silhouette is an epipolar line. A rich literature exists on exploiting epipolar tangents, both for orthographic cameras [7], [9], [14], [15] and perspective cameras [16], [17], [18], [19]. In particular, the works of [17] and [18] use only the two outermost epipolar tangents, which eliminates the need for matching corresponding epipolar tangents across different images. Although these methods have given good results, their main drawback is the limited number of epipolar tangency points per pair of images, generally only two: one at the top and one at the bottom of the silhouette. When additional epipolar tangency points are available, the goal is to match them across different views and handle their visibility, as proposed in [15] and [19]. An additional limitation of all these methods is their inability to cope with partial or truncated silhouettes, as in the examples shown in Fig. 1. 
(a)
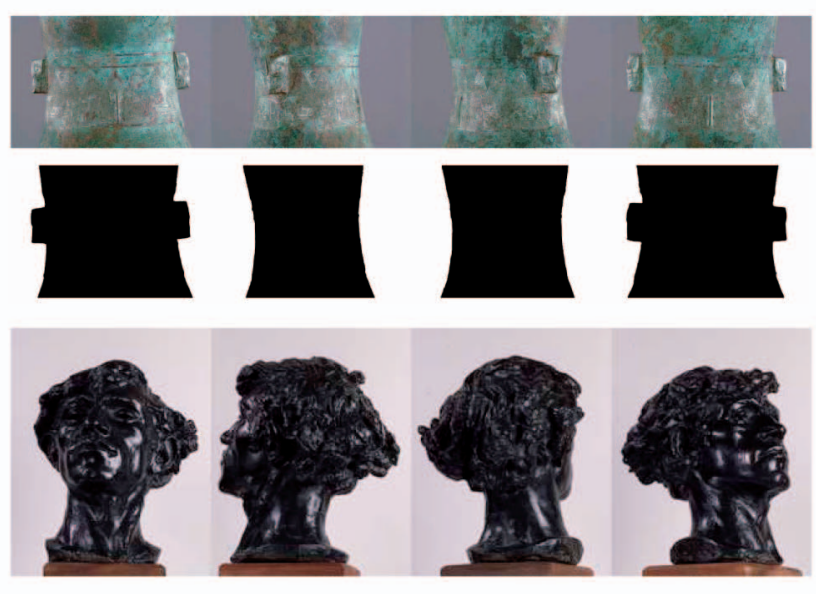

(b)

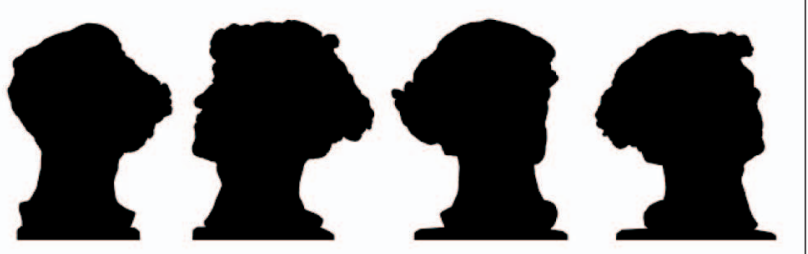

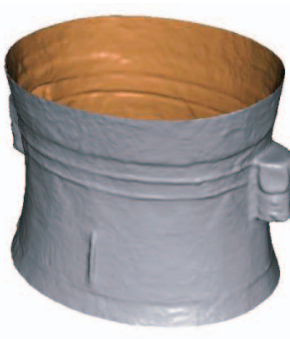

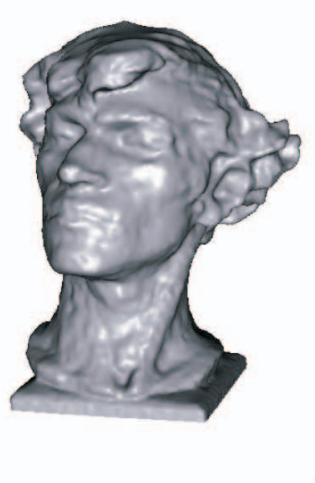

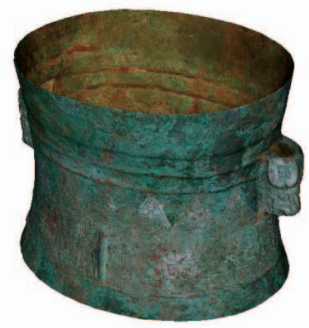

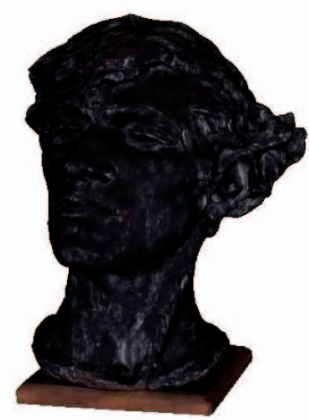

Fig. 1. Reconstructed sculptures after camera motion and focal length estimation using silhouette coherence. (a) Detail of a Chinese bronze vase (24 input images of 6 Mpixels, C2RMF, Paris). (b) Giganti by Camille Claudel (36 input images of 6 Mpixels, Cherbourg museum). Left bottom: corresponding segmented silhouettes. Middle: reconstructed shaded model. Right: textured model.

Although the notion of silhouette coherence appears in the literature under different names, it has never been exploited before for camera estimation. Bottino and Laurentini study the problem of silhouette compatibility in [20] for the case of orthographic projection, and give some rules to determine if a set of silhouettes can correspond to a real object. They do not provide a way to quantify the amount of incompatibility. In his PhD thesis, Cheung [21] used the phrase consistent alignment for the idealized registration of two visual hulls. However, in practice, his proposal was not feasible in an optimization algorithm because it was too computationally expensive. In the very recent work of [22], a similar concept to the silhouette coherence is described, but the authors discard using it in an optimization algorithm because of their discrete implementation. Silhouette coherence is also related to the work of [23], where both silhouette segmentation and camera motion can be computed from a sequence of images by minimizing the mismatch between the silhouettes and the projections of the reconstructed shape. However, the proposed approach requires a very good initialization and is computationally very expensive.

In this paper, we further develop the concept of silhouette coherence and link it to the epipolar geometry, and specifically to the tangency criterion as used by Wong and Cipolla [18]. In particular, the epipolar tangency criterion can be seen as a measure of silhouette coherence for the special case of only two silhouettes. When using more than two silhouettes, the proposed silhouette coherence extends the epipolar tangency criterion by exploiting all the information contained in the contours of the silhouettes, not just at the epipolar tangency points. This enables us to estimate the motion and the focal length correctly even when epipolar tangents are difficult to match or noisy (see Fig. 1a). The proposed silhouette coherence criterion is also related to [24], where silhouette coherence is used to register a laser model with a set of images. The main difference with this paper is that we do not require a $3 \mathrm{D}$ representation of the object in order to perform camera calibration. The object is implicitly reconstructed from the silhouettes by a visual hull method at the same time as the cameras are calibrated.

\section{Problem Formulation}

We consider a perspective projection camera model where the relation between a $3 \mathrm{D}$ point $\mathbf{M}$ and its $2 \mathrm{D}$ projection $\mathbf{m}$ is fully represented by the $3 \times 4$ camera projection matrix $\mathrm{P}$, where $\mathbf{m} \simeq$ $\mathrm{PM} \simeq \mathrm{K}[\mathrm{R} \mid \mathbf{t}] \mathbf{M}$ and the $3 \times 3$ rotation matrix $\mathrm{R}$ and the vector $\mathbf{t}$ represent the orientation and translation defining the pose of the camera. The calibration matrix $\mathrm{K}$ contains the intrinsic parameters of the camera. The aspect ratio and the skew factor are assumed to be known or ideal for our CMOS and CCD cameras; the only intrinsic parameters that we consider are the focal length $f$ (in pixels) and the principal point $\left(u_{0}, v_{0}\right)^{\top}$. Furthermore, since the effect of the translation $\mathbf{t}$ and the principal point $\left(u_{0}, v_{0}\right)^{\top}$ is very similar under the assumption of circular motion, the principal point is considered to simply be the center of the image.

For $n$ views, we parameterize the circular motion with $n+3$ parameters $^{1}$ (see Fig. 2): The spherical coordinates of the rotation axis $\left(\theta_{\mathbf{a}}, \phi_{\mathbf{a}}\right)$, the translation direction angle $\alpha_{\mathbf{t}}$, the $n-1$ camera angle steps $\Delta \omega_{i}$, and the focal length $f$. The $i$ th camera projection matrix $\mathrm{P}_{i}$ has the following decomposition:

$$
\mathrm{P}_{i}=\mathrm{K}\left[\mathrm{R}_{i} \mid \mathbf{t}_{i}\right]=\mathrm{K}\left[\mathrm{R}_{\mathbf{a}}\left(\omega_{i}\right) \mid \mathbf{t}\right] \forall i,
$$

where $R_{\mathbf{a}}\left(\omega_{i}\right)$ is the rotation of angle $\omega_{i}$ around axis $\mathbf{a}$ and $\mathbf{t}=\left(\sin \left(\alpha_{\mathbf{t}}\right), 0, \cos \left(\alpha_{\mathbf{t}}\right)\right)^{\top}$.

Given a set of silhouettes $S_{i}$ of a rigid object taken under circular motion (see Fig. 2a), our goal is to recover the corresponding projection matrices $\mathrm{P}_{i}$ as the set of $n+3$ parameters $\mathbf{v}=$ $\left(\theta_{\mathbf{a}}, \phi_{\mathbf{a}}, \alpha_{\mathbf{t}}, \Delta \omega_{i}, f\right)$ (see Fig. 2b).

\section{Silhouette Coherence}

Given a set of silhouettes $S_{i}$ of the same 3D object, taken from different points of view, and a corresponding set of camera projection matrices $\mathrm{P}_{i}$, we would like to measure the agreement of both the silhouette segmentation and the camera projection

1. We could also have used the parameterization of [11] instead. 
(a)

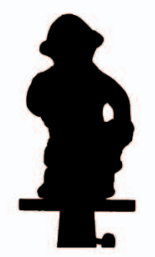

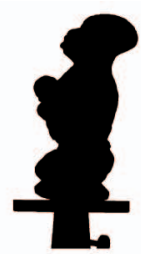
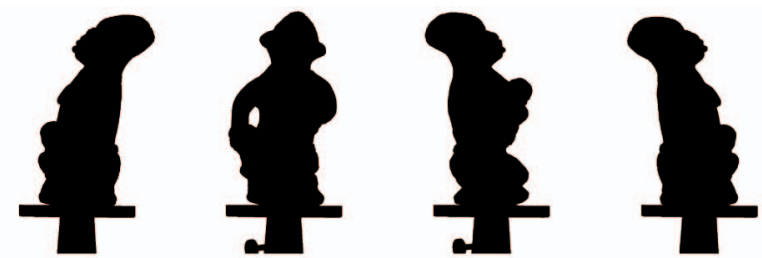

(b)

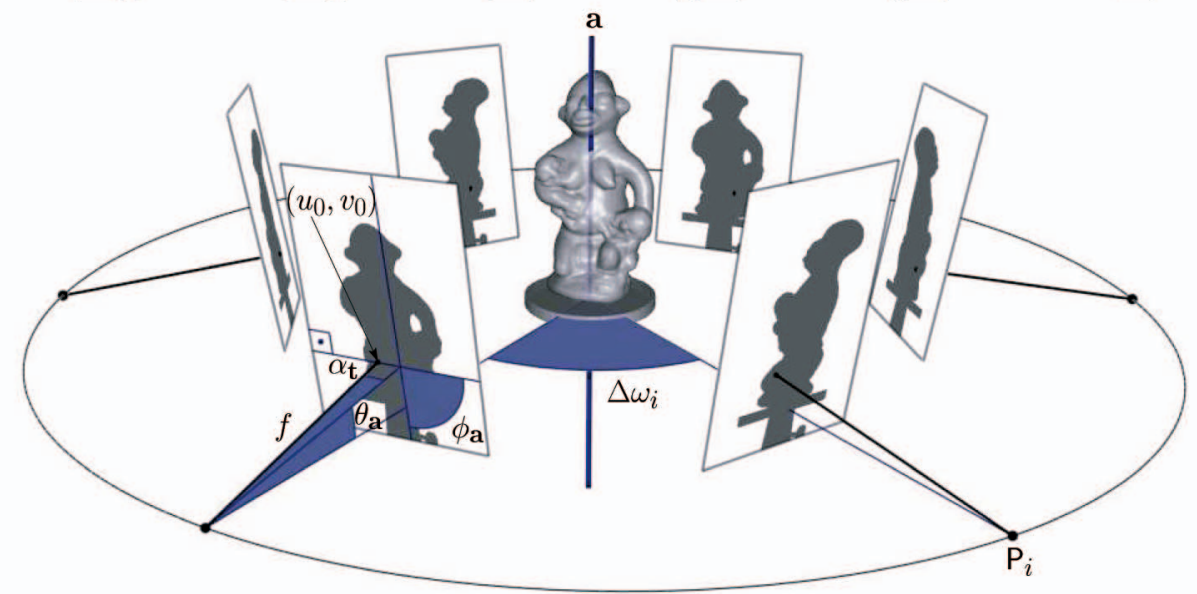

Fig. 2. Circular motion parameterization. (a) Set of input silhouettes $S_{i}$. (b) Parameterization of the projection matrices $\mathrm{P}_{i}$ as a function of the spherical coordinates of the rotation axis $\left(\theta_{\mathbf{a}}, \phi_{\mathbf{a}}\right)$, the translation direction $\alpha_{\mathbf{t}}$, the camera angle steps $\Delta \omega_{i}$, and the focal length $f$.

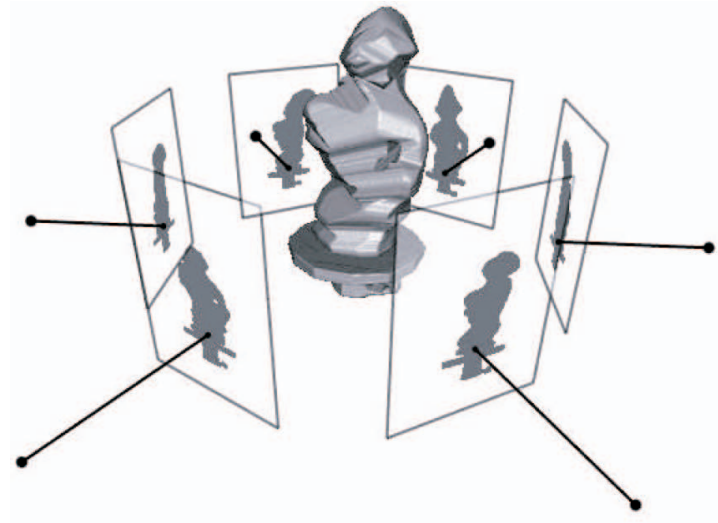

(a)

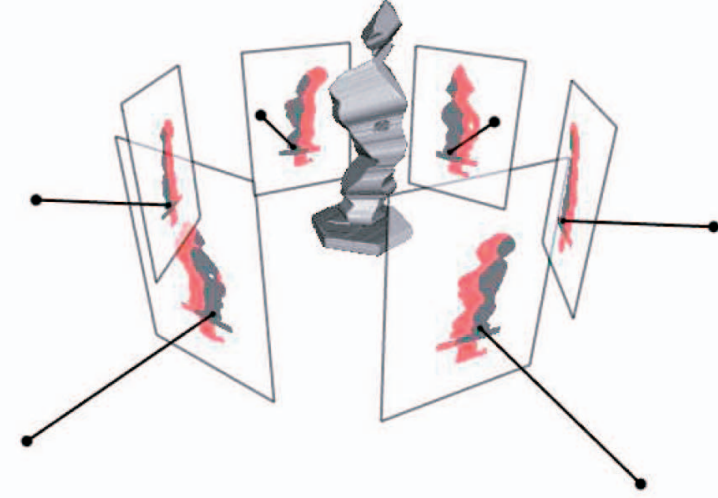

(b)

Fig. 3. Two examples of different degrees of silhouette coherence. The reconstructed visual hull $\mathcal{V}$ is shown by the gray 3D object. (a) A perfectly coherent silhouette set. (b) Same set of silhouettes with a different pose and low silhouette coherence. The red area shows noncoherent silhouette pixels. In this paper, we minimize the red area as a criterion for camera calibration.

matrices. We want to exploit the raw information provided by a silhouette: a binary classification of all the optic rays going through the optic center of the associated camera. These optic rays are labeled by the silhouette as intersecting the object (S label) if they belong to a silhouette pixel, or not intersecting the object (B label) if they belong to a background pixel.

Let us consider an optic ray defined by a silhouette pixel and thus classified as $\mathbf{S}$. The projection of the optic ray into any other view must intersect the corresponding silhouette. Furthermore, the back projection of all these 2D intersection intervals onto the optic ray must be coherent, meaning that their intersection must have nonzero length. The intersection interval will contain the exact position where the optic ray touches or intersects the object.

Due to noisy silhouettes or incorrect camera projection matrices, the above statement may not be satisfied, i.e., even if a silhouette has labeled an optic ray as $\mathbf{S}$, its depth interval might be empty. In the case of only two views, the corresponding silhouettes will not be coherent if there exists at least one optic ray classified as $\mathbf{S}$ by one of the silhouettes whose projection does not intersect the other silhouette. In the case of $n$ views, the lack of coherence is defined by the existence of at least one optic ray where the depth intervals defined by the $n-1$ other silhouettes have an empty intersection. This lack of coherence can be measured simply by counting how many optic rays in each silhouette are not coherent with the other silhouettes. Two examples of coherent and noncoherent silhouettes are shown in Fig. 3. The silhouette pixels that are not coherent with the other silhouettes are shown in red in Fig. 3b.

A simple way of measuring the silhouette coherence using the concept of visual hull [25] is as follows:

- compute the reconstructed visual hull defined by the silhouettes and the projection matrices,

- project the reconstructed visual hull back into the cameras, and

- compare the reconstructed visual hull silhouettes with the original silhouettes.

In the situation of ideal data, i.e., perfect segmentation and exact projection matrices, the reconstructed visual hull silhouettes and the 


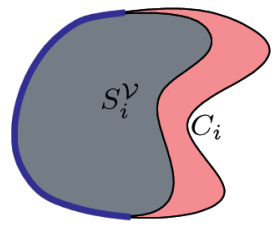

(a)

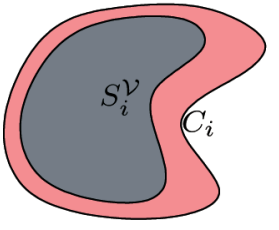

(b)

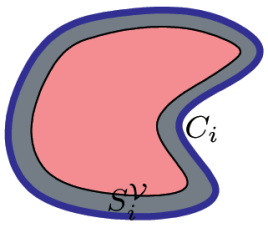

(c)
Fig. 4. Limitation of using contours for silhouette comparison. The silhouette of the visual hull $S_{i}^{\mathcal{V}}$ is shown in dark gray, the difference with $S_{i}$ is shown in red, and the intersection $C_{i} \cap S_{i}^{\mathcal{V}}$ is drawn with a thick blue stroke. (a) Ideal scenario: using contours and areas is equivalent. (b) Problematic case: the coherence using contours is much lower ( 0 in the example) than with areas. (c) Problematic case with a hole in the silhouette $S_{i}^{\mathcal{V}}$ : the coherence using contours is much higher (1 in the example) than with areas.

original silhouettes will be exactly the same (see Fig. 3a). With real data, both the silhouettes and the projection matrices will be imperfect. As a consequence, the original silhouettes and the reconstructed visual hull silhouettes will not be the same, the latter silhouettes being always contained in the original ones (see Fig. 3b).

\subsection{A Robust Measure of Silhouette Coherence}

Let $\mathcal{V}$ be the visual hull defined by the set of silhouettes $S_{i}$ and the set of projection matrices $\mathrm{P}_{i}$, and $S_{i}^{\mathcal{V}}$ its projection into the $i$ th image. A choice must be made about how to measure the similarity $\mathcal{C}$ between the silhouette $S_{i}$ and the projection of the reconstructed visual hull $S_{i}^{\mathcal{V}}$. A quick answer would be to use the ratio of areas between these two silhouettes as in [24]:

$$
\mathcal{C}\left(S_{i}, S_{i}^{\mathcal{V}}\right)=\frac{\int S_{i}^{\mathcal{V}}}{\int S_{i}}=\frac{\int\left(S_{i} \cap S_{i}^{\mathcal{V}}\right)}{\int S_{i}} \in[0,1] .
$$

However, this measure has the major disadvantage of a very high computation cost, as mentioned by [21]. To address this important issue, we propose a simple replacement in (2) of the silhouette $S_{i}$ by its contour, $C_{i}$ :

$$
\mathcal{C}\left(S_{i}, S_{i}^{\mathcal{V}}\right)=\frac{\int\left(C_{i} \cap S_{i}^{\mathcal{V}}\right)}{\int C_{i}} \in[0,1] .
$$

This new measure is much faster than (2) since, as discussed in Section 5, we propose to discretize the evaluation of the measure. Hence, the computation time of (3) is proportional to the length term $\left(C_{i} \cap S_{i}^{\mathcal{V}}\right)$, while the computation time of (2) is proportional to the area term $\left(S_{i} \cap S_{i}^{\mathcal{V}}\right)$. However, a possible weakness concerning the use of the contour instead of the silhouette itself is that these two measures might differ for some problematic cases as shown in Fig. $4 \mathrm{~b}$ and Fig. $4 \mathrm{c}$. The contour-based measure will penalize the Fig. $4 \mathrm{~b}$ scenario, while encouraging scenarios such as Fig. $4 \mathrm{c}$. If none of the silhouettes has interior holes, the latter is impossible by virtue of how the visual hull is constructed. Case b, however, is much more common for the problem of silhouette coherence and can be easily reproduced if one of the silhouettes is dilated due to a segmentation error. In order to alleviate this limitation, we propose a $\delta$-offset silhouette contour approach (see Fig. 5). For a given $\delta$ value, we replace in (3) the contour $C_{i}$ by its eroded version of $\delta$ pixels $C_{i} \ominus \delta$, which gives:

$$
\mathcal{C}^{\delta}\left(S_{i}, S_{i}^{\mathcal{V}}\right)=\frac{\int\left(\left(C_{i} \ominus \delta\right) \cap S_{i}^{\mathcal{V}}\right)}{\int\left(C_{i} \ominus \delta\right)} \in[0,1] .
$$

Increasing $\delta$ makes the new measure more robust against bad segmentation. But robustness is obtained at the price of accuracy. For a given $\delta$ value, the silhouette coherence will not be able to distinguish between a silhouette $S_{i}$ and its reconstructed visual

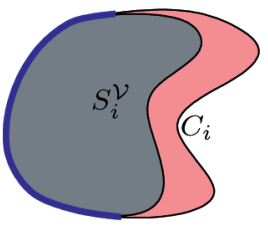

(a)

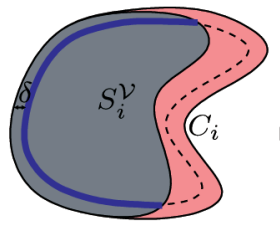

(b)

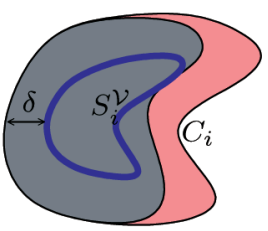

(c)
Fig. 5. Avoiding the limitation of using contours for silhouette comparison. From left to right, increasing $\delta$ values imply increasing silhouette coherence values and better robustness for scenario of Fig. $4 \mathrm{~b}$. The original silhouette $S_{i}$ corresponds to the outermost contour. The silhouette of the reconstructed visual hull $S_{i}^{\mathcal{V}}$ is shown in dark gray. The term $\left(C_{i} \ominus \delta\right) \cap S_{i}^{\mathcal{V}}$ in (4) is shown with a thick blue stroke.

hull silhouette $S_{i}^{\mathcal{V}}$ if their difference is smaller than $\delta$. Typical values of $\delta$ range from 0.25 pixels to several pixels, depending on the quality of the silhouette segmentation.

Equation (4) evaluates the coherence between the silhouette $S_{i}$ and all the other silhouettes $S_{j \neq i}$ that contributed to the reconstructed visual hull. In fact, since $S_{i}^{\mathcal{V}}$ is fully determined by the silhouette contours $C_{j=1, \cdots, n}$, (4) can also be noted as $\mathcal{C}^{\delta}\left(C_{i}, C_{j=1, \cdots, n}\right)$, or $\mathcal{C}^{\delta}\left(C_{i}, C_{j \neq i}\right)$. To compute the total coherence between all the silhouettes, we simply compute the average coherence between each silhouette and the $n-1$ others:

$$
\mathcal{C}^{\delta}\left(C_{1}, \ldots, C_{n}\right)=\frac{1}{n} \sum_{i=1}^{n} \mathcal{C}^{\delta}\left(C_{i}, C_{j \neq i}\right) \in[0,1] .
$$

\subsection{Relation to Epipolar Geometry and Epipolar Tangents}

The proposed silhouette coherence criterion can be seen as an extension to methods based on epipolar tangency points. For a given pair of views, as shown in Fig. 6, the epipolar tangency approach minimizes the square distance between epipolar tangents of one view $\left(l_{a}\right.$ and $l_{b}$ in view $i, l_{c}$ and $l_{d}$ in view $\left.j\right)$ and the transferred epipolar tangents of the other view via the fundamental matrix $\mathrm{F}_{i j}=\left[e_{i j}\right]_{\times} \mathrm{H}_{\infty}\left(\mathrm{H}_{\infty}^{-\top} l_{c}\right.$ and $\mathrm{H}_{\infty}^{-\top} l_{d}$ in view $i$, $\mathrm{H}_{\infty}^{\top} l_{a}$ and $\mathrm{H}_{\infty}^{\top} l_{b}$ in view $j$ ). That is, it minimizes the sum of geometric distances $\mathcal{C}_{e t}\left(C_{i}, C_{j}\right)=d_{a c}^{2}+d_{b d}^{2}+d_{c a}^{2}+d_{d b}^{2}$. For the same pair of silhouettes, the optimization of the coherence criterion corresponds to maximizing the lengths $C_{i} \cap S_{i}^{\mathcal{V}}$ and $C_{j} \cap S_{j}^{\mathcal{V}}$. So, we can see that, except for degenerate configurations, both criteria try to minimize the sectors defined by the epipolar tangents in one view and their corresponding epipolar tangents in the other view.
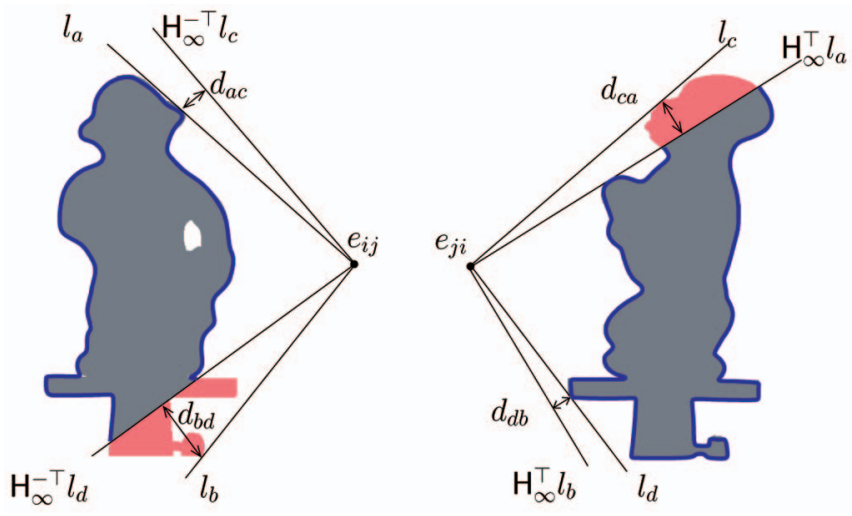

Fig. 6. Comparison of epipolar tangency and silhouette coherence criteria for $n=2$ silhouettes. The silhouettes of the visual hull $S_{i}^{\mathcal{V}}$ and $S_{j}^{\mathcal{V}}$ are shown in dark gray. The terms $C_{i} \cap S_{i}^{\mathcal{V}}$ and $C_{j} \cap S_{j}^{\mathcal{V}}$ are drawn with a thick blue stroke. For the case of two views, both criteria attempt to minimize the sectors defined by $l_{b}$ and $\mathrm{H}_{\infty}^{-\top} l_{d}$, and $l_{c}$ and $\mathrm{H}_{\infty}^{\top} l_{a}$ (shown in red). 


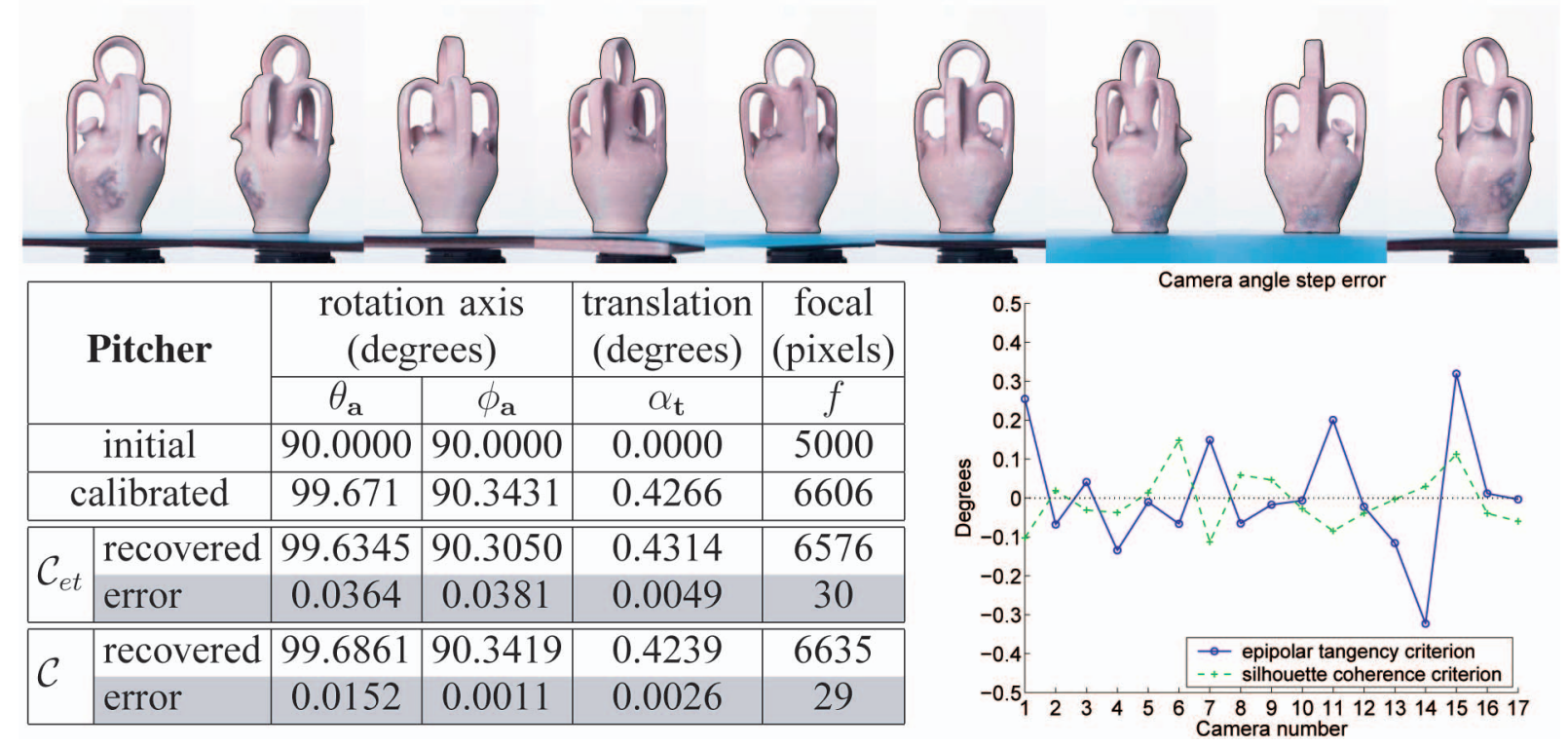

Fig. 7. Camera estimation for the Pitcher sequence. Top: Some of the original images superimposed with the extracted smooth polygon contours (in black). Bottom: Camera estimation results. Mean camera angle step error of 0.11 degrees using epipolar tangency points $\left(\mathcal{C}_{e t}\right)$ and 0.06 degrees using silhouette coherence $(\mathcal{C})$. Note that the axis error is reduced by a factor of 3 , the translation error by a factor of 2 , and the step error by a factor of 2 .

Thus, if we optimize our coherence criterion taking the silhouettes pairwise, we get the same behavior as with methods based on epipolar tangents, e.g., [18].

When using the proposed silhouette coherence for $n>2$, silhouettes are not just taken pairwise but all at the same time. This means that the information we exploit is not just at the epipolar tangency points but all over the silhouette contour. As a result, even if we use silhouettes where the outer epipolar tangents are not available, the silhouette coherence criterion is still valid. We present an example in Fig. 1a, where we do not have the top and the bottom of the silhouettes (no outer epipolar tangents available) but for which we are still able to estimate the motion and the focal length with very good accuracy.

It is worth noting that, as pointed out by [21], maximizing silhouette coherence is a necessary condition but not a sufficient one in order to recover camera motion. The two main causes for this ambiguity are the object shape and the number of silhouettes. A sphere turning around its center is an example of shape ambiguity. All the silhouettes are the same and, therefore, there is no unique solution to the problem of silhouette coherence maximization. Thus, in general, we cannot guarantee the uniqueness of the solution. Also, if we use only a small number of silhouettes, then the silhouette coherence may be maximized for a large class of solutions. However, we can expect that, if we take a sufficient number of pictures, real objects are generally asymmetric enough to guarantee a unique solution as shown by the practical examples.

Moreover, since silhouette coherence is an extension of epipolar tangency criteria, the same limitation applies to previous methods using epipolar tangency points. If silhouette coherence is optimized, so is the epipolar tangency criterion. This can be checked easily in Fig. 6. In practice, maximizing silhouette coherence is sufficient and can be used for camera calibration, as demonstrated by the reconstruction of more than 50 sequences (available for download at [26]) obtained using the 3D modeling technique described in [2]. In order to use the modeling algorithm, cameras were calibrated using the technique described in this paper.

\section{Overview of the Camera Estimation ALGORITHM}

We now present a practical implementation of the silhouette coherence criterion $\mathcal{C}^{\delta}$, achieved by discretizing the contour $C_{i} \ominus \delta$ into a number of equally spaced sample points. The term $\left(C_{i} \ominus \delta\right) \cap$ $S_{i}^{\mathcal{V}}$ is evaluated by testing, for each sample point, if its associated optic ray intersects the reconstructed visual hull using a ray casting technique [27]. A simplified version of this algorithm is used, where we do not take into account contours inside the silhouettes. Furthermore, we do not compute all the depth intervals for a given optic ray. We just compute the minimum and maximum of the interval intersection with each silhouette. This is a conservative approximation of the real coherence, i.e., the coherence score that we obtain by storing only the minimum and maximum depths is always equal or greater than the real one. However, in practice, the deviation from the coherence computed with all the intervals is small. The algorithm describing the silhouette coherence $\mathcal{C}^{\delta}\left(C_{i}, C_{j \neq i}\right)$ between a given silhouette contour $C_{i}$ and the remaining silhouette contours $C_{j \neq i}$ is shown in Algorithm 3. If $N$ is the number of sample points per silhouette, and $n$ is the number of silhouettes, the complexity of $\mathcal{C}^{\delta}\left(C_{i}, C_{j \neq i}\right)$ is $\mathcal{O}(n N \log (N))$. The total silhouette coherence $\mathcal{C}^{\delta}\left(C_{i}, \ldots, C_{n}\right)$ in (5) is shown in Algorithm 2 and its complexity is $\mathcal{O}\left(n^{2} N \log (N)\right)$. As an example, the computation time of one evaluation of (5) on an Athlon 1.5 GHz processor is $750 \mathrm{~ms}$ for the Pitcher example of Fig. 7 ( $n=18, N \approx 6,000$ ).

In order to exploit silhouette coherence for camera motion and focal length estimation under circular motion, the key is to use the silhouette coherence as the cost in an optimization procedure. Equation (5) can be seen as a "black box" that takes as input a set of silhouettes and projection matrices, and gives as output a scalar value of silhouette coherence. We use Powell's derivative-free optimization algorithm [28] to maximize (5). Several hundred cost evaluations are typically required before convergence. The system is always initialized with the same default circular motion: the rotation axis $\mathbf{a}=(0,1,0)^{\top}\left(\theta_{\mathbf{a}}=\frac{\pi}{2}, \phi_{\mathbf{a}}=\frac{\pi}{2}\right)$, the translation $\mathbf{t}=$ $(0,0,1)^{\top}\left(\alpha_{\mathbf{t}}=0\right)$, and the initial guess of the camera angles (e.g., $\left.\Delta \omega_{i}=\frac{2 \pi}{n}\right)$. The initial guess of the focal length $f_{0}$ is directly 
computed from typical values of the field of view, e.g., 20 degrees. The principal point is considered constant and equal to the center of the image. The complete algorithm for motion and focal length estimation is described in Algorithm 1. Because circular motion is a very constrained motion, we have found that the initial values for the rotation axis, the translation direction and the focal length do not need to be very close to the actual solution. The only source of convergence problems is the initial camera angles, but the algorithm has proven to have good convergence properties for camera angle errors of up to 15 degrees.

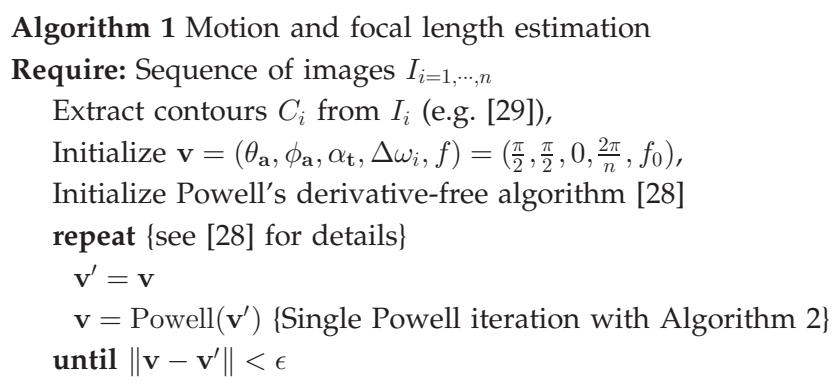

Algorithm 2 Total silhouette coherence $\mathcal{C}^{\delta}\left(C_{i=1, \cdots, n}\right)$

Require: Sequence of contours $C_{i=1, \cdots, n}$, parameters $\mathbf{v}=\left(\theta_{\mathbf{a}}, \phi_{\mathbf{a}}, \alpha_{\mathbf{t}}, \Delta \omega_{i}, f\right)$

Compute $\mathrm{P}_{i}$ defined by v (see Fig. $2 \mathrm{~b}$ )

Return average $\frac{1}{n} \sum_{i=1}^{n} \mathcal{C}^{\delta}\left(C_{i}, C_{j \neq i}\right)$ \{Algorithm 3\}

Algorithm 3 Silhouette coherence $\mathcal{C}^{\delta}\left(C_{i}, C_{j \neq i}\right)$

Require: Projection matrices $\mathrm{P}_{i} \forall i$, reference contour $C_{i}$, contour list $C_{j \neq i}$, contour offset $\delta$, number of samples per contour $N$

Build point list $\mathbf{m}^{(k)}$, sampling $N$ points along $C_{i} \ominus \delta$

for all $\mathbf{m}^{(k)}$ do

Initialize 3D interval $I_{3 D}=[0, \infty]$

Initialize counter $N^{\prime}=0$

for all $C_{j \neq i}$ do

Project optic ray $l=\mathrm{P}_{j} \mathrm{P}_{i}^{-1} \mathbf{m}^{(k)}$

Compute 2D intersection interval $I_{2 D}=l \cap C_{j}$ Back project 2D interval $I_{3 D}=I_{3 D} \cap \mathrm{P}_{j}^{-1} I_{2 D}$

\section{end for}

$$
\begin{aligned}
& \text { if } I_{3 D} \neq \emptyset \text { then } \\
& \qquad N^{\prime}=N^{\prime}+1 \\
& \text { end if }
\end{aligned}
$$

end for

Return $\frac{N^{\prime}}{N}$

\section{EXPERIMENTAL RESULTS}

We present an experiment using a Pitcher sequence with 18 color images of 2,008 $\times 3,040$ pixels acquired with a computer controlled turntable. The images have been segmented by an automatic procedure [29] (see the top of Fig. 7). For evaluation, we also use a sequence of a calibration pattern in order to accurately recover the intrinsic parameters and the circular motion using [30]. The $\delta$-offset used for the silhouette coherence criterion is $\delta=0.25$ pixels due to the subpixel accuracy of the silhouette extraction. The camera angles are initialized with a uniform random noise in the interval $[-15,15]$ degrees around the true angles.

Fig. 7 contains the results for the camera motion (rotation axis, translation direction, and camera angles) and focal length estimation problem. A total of 21 parameters are recovered. We compare the proposed silhouette coherence with the state-of-theart method described in [18]. The silhouette coherence clearly outperforms [18] by reducing the rotation axis error by a factor of 3 , the translation error by a factor of 2, and the camera angles error by a factor of 2 . Both criteria recover the focal length with the same accuracy $(\sim 0.5$ percent).

The same camera estimation algorithm has been repeatedly tested successfully on over 50 uncalibrated sequences. We illustrate in Fig. 1 two of these sequences that are particularly interesting. For the Chinese bronze vase (Fig. 1a), the two outermost epipolar tangents are not available, since the tops and bottoms of the silhouettes are truncated. For the Giganti sculpture (Fig. 1b), just the bottom has been truncated. Problems with correctly extracting the bottom of an object are common under turntable motion. In general, it is easy to extract the top of an object, but it is much more difficult to separate the bottom from the turntable. Without any ground truth for the camera parameters, we validate the camera estimation results by the visual quality of the final reconstructions. They have been obtained by using the estimated camera parameters together with the 3D modeling technique described in [2].

Note that the Giganti sculpture (Fig. 1b) would be very difficult to calibrate using point-based techniques, its surface being very specular, while the Chinese vase (Fig. 1a) is impossible for epipolar tangent algorithms.

Two additional experiments ara available as an appendix of a technical report [31]. In the first experiment, we compare the accuracy of the silhouette coherence and the epipolar tangency criteria as a function of silhouette noise. In the second experiment, we show that silhouette coherence exploits more information that epipolar tangency points alone by showing that it can calibrate the cameras (up to a certain ambiguity) even when no epipolar tangency points are available.

\section{Conclusions AND FUtURe Work}

A new approach to silhouette-based camera estimation has been developed. It is built on the concept of silhouette coherence, defined as a similarity between a set of silhouettes and the silhouettes of their visual hull. This approach has been successfully tested for the problem of circular motion and validated both qualitatively and quantitatively. The high accuracy of the results is due to the use of the full silhouette contour in the computation, whereas previous silhouette-based methods just use epipolar tangency points. The proposed method eliminates the need for epipolar tangency points and naturally copes with truncated silhouettes.

A limitation of our current silhouette coherence implementation is the discretization of the silhouette contours. To remove this source of sampling noise, a solution would compute the exact visual hull silhouettes as polygons and compare them with the original silhouettes. To compute the exact silhouette of the visual hull, we can proceed as in [32], using a ray casting technique.

We are currently extending the proposed approach to roughly circular motion and general motion, but special attention has to be paid to the initialization process to avoid local minima, less important for the case of circular motion.

\section{ACKNOWLEDGMENTS}

The authors would like to thank Peter Sturm and Gabriel Brostow for their comments about this work.

\section{REFERENCES}

[1] K.N. Kutulakos and S.M. Seitz, "A Theory of Shape by Space Carving," Int'l J. Computer Vision, vol. 38, no. 3, pp. 199-218, 2000. 
[2] C. Hernández and F. Schmitt, "Silhouette and Stereo Fusion for 3D Object Modeling," Computer Vision and Image Understanding, vol. 96, no. 3, pp. 367392, 2004.

[3] G. Vogiatzis, P. Torr, and R. Cipolla, "Multi-View Stereo via Volumetric Graph-Cuts," Proc. IEEE CS Conf. Computer Vision and Pattern Recognition, pp. 391-398, 2005.

[4] P. Gargallo and P. Sturm, "Bayesian 3D Modeling from Images Using Multiple Depth Maps," Proc. IEEE CS Conf. Computer Vision and Pattern Recognition, vol. II, pp. 885-891, 2005.

[5] R.I. Hartley and A. Zisserman, Multiple View Geometry in Computer Vision. Cambridge Univ. Press, 2000.

[6] D. Nistér, "An Efficient Solution to the Five-Point Relative Pose Problem," IEEE Trans. Pattern Analysis and Machine Intelligence, vol. 26, no. 6, pp. 756770, June 2004.

[7] J. Rieger, "Three Dimensional Motion from Fixed Points of a Deforming Profile Curve," Optics Letters, vol. 11, no. 3, pp. 123-125, 1986.

[8] J. Porrill and S.B. Pollard, "Curve Matching and Stereo Calibration," Image and Vision Computing, vol. 9, no. 1, pp. 45-50, 1991.

[9] P. Giblin, F. Pollick, and J. Rycroft, "Recovery of an Unknown Axis of Rotation from the Profiles of a Rotating Surface," J. Optical Soc. Am. vol. 11A, pp. 1976-1984, 1994.

[10] R. Cipolla and P. Giblin, Visual Motion of Curves and Surfaces. Cambridge Univ. Press, 2000.

[11] A. Fitzgibbon, G. Cross, and A. Zisserman, "Automatic 3D Model Construction for Turn-Table Sequences," 3D SMILE, pp. 155-170, 1998.

[12] G. Jiang, H. Tsui, L. Quan, and A. Zisserman, "Single Axis Geometry by Fitting Conics," Proc. European Conf. Computer Vision, vol. 1, pp. 537-550, 2002.

[13] R. Cipolla, K. Åström, and P. Giblin, "Motion from the Frontier of Curved Surfaces," Proc. Int'l Conf. Computer Vision, pp. 269-275, June 1995.

[14] B. Vijayakumar, D. Kriegman, and J. Ponce, "Structure and Motion of Curved 3d Objects from Monocular Silhouettes," Proc. IEEE CS Conf. Computer Vision and Pattern Recogni, pp. 327-334, 1996.

[15] Y. Furukawa, A. Sethi, J. Ponce, and D. Kriegman, "Structure and Motion from Images of Smooth Textureless Objects," Proc. European Conf. Computer Vision 2004, vol. 2, pp. 287-298, May 2004.
[16] K. Åström, R. Cipolla, and P. Giblin, "Generalized Epipolar Constraints," Int'l J. Computer Vision, vol. 33, no. 1, pp. 51-72, 1999.

[17] P.R.S. Mendonça, K.-Y.K. Wong, and R. Cipolla, "Epipolar Geometry from Profiles under Circular Motion," IEEE Trans. Pattern Analysis and Machine Intelligence, vol. 23, no. 6, pp. 604-616, June 2001.

[18] K.-Y. K. Wong and R. Cipolla, "Reconstruction of Sculpture from Its Profiles with Unknown Camera Positions," IEEE Trans. Image Processing, vol. 13, no. 3, pp. 381-389, 2004.

[19] S.N. Sinha, M. Pollefeys, and L. McMillan, "Camera Network Calibration from Dynamic Silhouettes," Proc. IEEE CS Conf. Computer Vision and Pattern Recogni, vol. 1, pp. 195-202, 2004.

[20] A. Bottino and A. Laurentini, "Introducing a New Problem: Shape-fromSilhouette When the Relative Positions of the Viewpoints Is Unknown," IEEE Trans. Pattern Analysis and Machine Intelligence, vol. 25, no. 11, pp. 1484-1493, Nov. 2003.

[21] K. Cheung, "Visual Hull Construction, Alignment, and Refinement for Human Kinematic Modeling, Motion Tracking, and Rendering," PhD dissertation, Carnegie Mellon Univ., 2003.

[22] E. Boyer, "On Using Silhouettes for Camera Calibration," Proc. Asian Conf. Computer Vision, pp. 1-10, 2006.

[23] A.J. Yezzi and S. Soatto, "Structure from Motion for Scenes without Features," Proc. IEEE CS Conf. Computer Vision and Pattern Recogni, vol. I, pp. 171-178, 2003.

[24] H. Lensch, W. Heidrich, and H.P. Seidel, "A Silhouette-Based Algorithm for Texture Registration and Stitching," J. Graphical Models, pp. 245-262, 2001.

[25] A. Laurentini, "The Visual Hull Concept for Silhouette Based Image Understanding," IEEE Trans. Pattern Analysis and Machine Intelligence, vol. 16, no. 2, Feb. 1994

[26] http://www.tsi.enst.fr/3dmodels/, 2006

[27] W. Matusik, C. Buehler, R. Raskar, S. Gortler, and L. McMillan, "ImageBased Visual Hulls," Proc. ACM SIGGRAPH, pp. 369-374, 2000.

[28] M. Powell, "An Efficient Method for Finding the Minimum of a Function of Several Variables without Calculating Derivatives," Computer J., vol. 17, pp. 155-162, 1964.

[29] C. Xu and J.L. Prince, "Snakes, Shapes, and Gradient Vector Flow," IEEE Trans. Image Processing, pp. 359-369, 1998.

[30] J.M. Lavest, M. Viala, and M. Dhome, "Do We Really Need an Accurate Calibration Pattern to Achieve a Reliable Camera Calibration," Proc. European Conf. Computer Vision, vol. 1, pp. 158-174, 1998.

[31] C. Hernández, F. Schmitt, and R. Cipolla, "Error Analysis of Silhouette Coherence for Camera Calibration Under Circular Motion," Technical Report 559, Univ. of Cambridge, June 2006.

[32] J.-S. Franco and E. Boyer, "Exact Polyhedral Visual Hulls," Proc. British Machine Vision Conf., pp. 329-338, Sept. 2003.

$\triangleright$ For more information on this or any other computing topic, please visit our Digital Library at www.computer.org/publications/dlib. 